\title{
Pflegegeldeinstufung durch die Pflege
}

\author{
Große Erwartungen an weitere Maßnahmen und Strukturänderungen
}

Vorerst einmal zufrieden mit dem Pilotprojekt zur Pflegegeldeinstufung durch Mediziner und Pflegepersonen gemeinsam zeigten sich die österreichischen Interessensvertretungen im Bereich Pflege. Allerdings betonen sie mehr oder minder vehement, dass dies nur ein erster Schritt sein könne. Die Vorteile die eine Beteiligung der Pflege in diesem Verfahren bringe, kommen sowohl den Pflegegeldbeziehern als auch dem Steuerzahler zugute, denn: Eine fachgerechte Einschätzung verhindere langwierige und kostenaufwändig Klagen der Betroffenen auf Höhereinstufung.

Die Verbesserung der Qualität im Zusammenhang mit der Einstufung und damit die Steigerung der Kundenzufriedenheit sieht die neugegründete ARGE Mobile Pflege im Österreichischen Gesundheits- und Krankenpflegeverband (ÖGKV) durch die Verlagerung der Einstufungsberechtigung an den Gehobenen Dienst der Gesundheits- und Krankenpflege. Die gesetzlichen Voraussetzungen liegen demnach schon mit dem Gesundheits- und Krankenpflegegesetz aus dem Jahr 1997 vor. Dort werde, so das Positionspapier vom vergangenen Sommer, in $\S 14$ die Eigenverantwortlichkeit klar definiert „und somit die Zuständigkeit des gehobenen Dienstes für Gesundheitsund Krankenpflege in Bezug auf die Erhebung der Pflegebedürfnisse und des Grades der Pflegeabhängigkeit der Pflegegeld-Werber, sowie die Feststellung und Beurteilung der zur Deckung dieser Bedürfnisse zur Verfügung stehenden Ressourcen (Pflegeanamnese)." Aus Sicht des ÖGKV sollte die Pflegegeldeinstufung dem gehobenen Dienst der Gesundheits- und Krankenpflege vorbehalten sein, „da der Bereich der Erhebung und Einschätzung des Pflegebedarfs dessen Kernkompetenz ist.“ Nur dann, wenn der Bedarf nach einer medizinischen Begutachtung auftritt, sollte die Einstufung gemeinsam mit dem Arzt durchgeführt werden bzw. die notwendigen Informationen eingeholt werden. Dabei sei die Einstufung des Pflegegeldes von einer unabhängigen Pflegeperson durchzuführen, die also nicht die zuständige Bezugspflegeperson sein kann.

Die Neuregelung der Pflegegeldeinstufung würde, so der ÖGKV, die dringend notwendige Entkoppelung von medizinischen Diagnosen erreichen und den tatsächlichen Pflege- und Betreuungsbedarf als Orientierung heranziehen. Nach diesem ersten Schritt des nun angelaufenen Projekts des Sozialministeriums zur gemeinsamen Einstufung durch Arzt und Pflegeperson sei es Ziel des ÖGKV, die Einstufung des Pflegebedarfs und der -bedürftigkeit als eigenverantwortliche Aufgabe der Profession Pflege anzuerkennen und dies entsprechend umzusetzen.

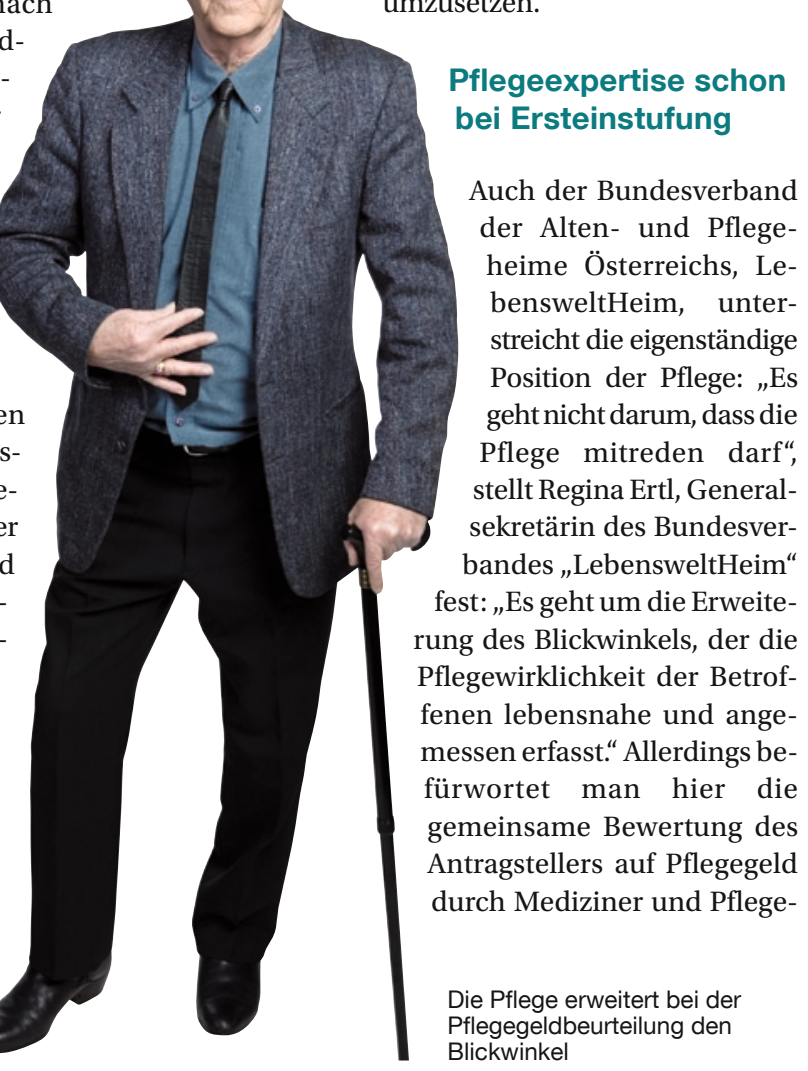

person und erwartet sich durch die multidisziplinäre Zusammenarbeit eine realistischere Feststellung des Aufwands im Pflegealltag und eine präzisere Einstufung. Da für die Pflegegeldeinstufung aber der Pflegeaufwand, der mit der medizinischen Diagnose verbunden ist, ausschlaggebend ist, fordert der Verband, dass bereits bei der ersten Einstufung die Pflegeexpertise Teil der Bewertung sein sollte.

\section{Abgestimmtes Gesamtpaket nötig}

Wenn auch der Schritt allgemein von den Interessenverbänden und Pflegeorganisationen begrüßt wird, so sehen alle dies nur als Beginn, dem weitere Reformen folgen müssen. Das Rote Kreuz fordert als Reaktion ein abgestimmtes Gesamtpaket für die Pflege. „Investitionen im Pflegebereich sind Investitionen in die Zukunft unserer Gesellschaft", erklärte Werner Kerschbaum, stv. Generalsekretär des Österreichischen Roten Kreuzes. „,Sachleistungen der Länder wie mobile Pflege, Pflegeheime und Tageseinrichtungen sowie Tarife sollen zukünftig harmonisiert, aber nicht nivelliert werden. Der Pflegebereich hat großes Wachstumspotential, das es zu nutzen gilt." Einzelmaßnahmen wie das Pilotprojekt zur Pflegegeldeinstufung seien nicht genug. Auch müssten Angehörige pflegebedürftiger Personen besser in den Einstufungsprozess eingebunden werden.

\section{Einstufungen harmonisieren}

Eine Anerkennung des Berufsstandes sieht die Volkshilfe in der Einbeziehung des Pflegepersonals bei der Einstufung des Pflegegelds. Dies werde auch zu einer Verbesserung der Situation pflege- und betreuungsbedürftiger Menschen führen. „Der Pilotversuch wird aber auch zeigen, wie unterschiedlich die Einstufungen in den fünf Testregionen vorgenommen werden und hoffentlich zu einer Harmonisierung führen“, so Volkshilfe Bundesgeschäftsführer Mag. (FH) Erich Fenninger. Darüberhinaus sei eine Valorisierung des Pflegegeldes, also eine Erhöhung entsprechend den gestiegenen Kosten, notwendig, fordern die Pflegeorganisationen.

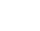

